\title{
АЛГОРИТМ ПОИСКА ПАРАМЕТРИЧЕСКИХ ДЕФЕКТОВ В НЕПРЕРЫВНЫХ САУ С ПОМОЩЬЮ НАСТРАИВАЕМЫХ МОДЕЛЕЙ НА ФИЛЬТРАХ ЛАГЕРРА 1
}

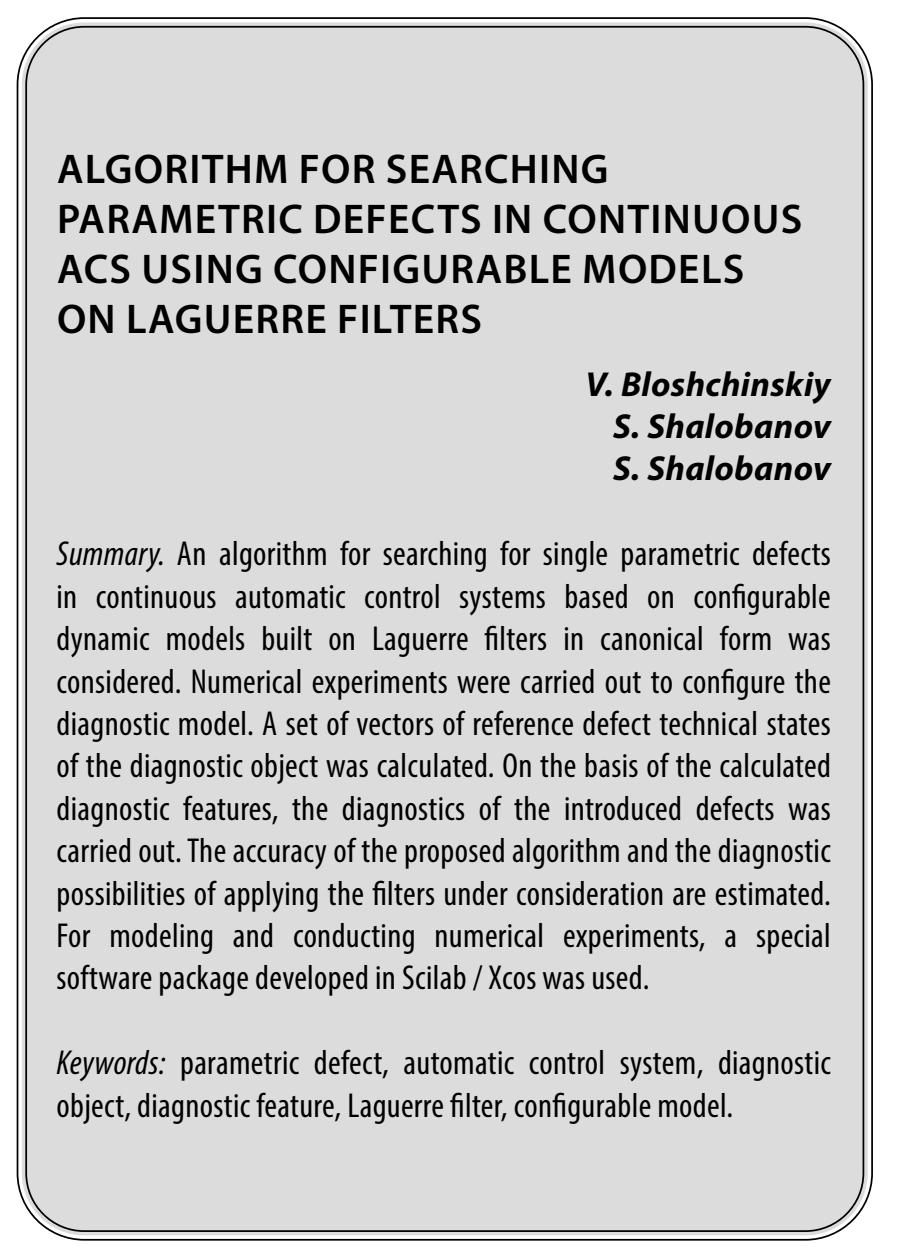

\section{Введение}

$\mathbf{H}$ а сегодняшний день вопросы диагностики и идентификации систем автоматического управления (САУ) остаются актуальными ввиду возрастающих требований к надежности и качеству таких систем. В данном ключе техническая диагностика САУ является активно развивающимся направлением, а задачи разработки и исследования алгоритмов диагностирования [1-4] занимают важное место в проблемах построения высокоэффективных методов ди-
Блощинский Владислав Дмитриевич

Аспирант, Тихоокеанский государственный университет, Хабаровск vladkms94@mail.ru

Шалобанов Сергей Викторович Д.т.н, профессор, Тихоокеанский государственный университет, Хабаровск shalobanov@mail.ru

Шалобанов Сергей Сергеевич

К.т.н, дочент, Тихоокеанский государственный университет, Хабаровск shalobanov_ne@mail.ru

Аннотация. Рассмотрен алгоритм поиска одиночных параметрических дефектов в непрерывных системах автоматического управления, основанный на настраиваемых динамических моделях, построенных на фильтрах Лагерра в канонической форме. Проведены численные эксперименты по настройке диагностической модели. Вычислен набор векторов эталонных дефектных технических состояний объекта диагностирования. На основе рассчитанных диагностических признаков осуществлено диагностирование вводимых дефектов. Дана оценка точности предлагаемого алгоритма и диагностических возможностей применения рассматриваемых фильтров. Для моделирования и проведения численных экспериментов использовался разработанный в Scilab/Xcos специальный программный комплекс.

Ключевые слова: параметрический дефект, система автоматического управления, объект диагностирования, диагностический признак, фильтр Лагерра, настраиваемая модель.

агностического обеспечения технических систем. При диагностировании технического состояния систем автоматического управления широкое применение находят традиционные типы математических моделей, которые являются предметом изучения теории идентификации [5-7].

Данная работа является логическим продолжением работ [8, 9], где была рассмотрена возможность применения динамических моделей, построенных на фильтрах на элементах задержки и фильтрах Лагерра, для

${ }^{1}$ Работа выполнена при поддержке гранта Президента Российской Федерации № МК-1616.2020.8 


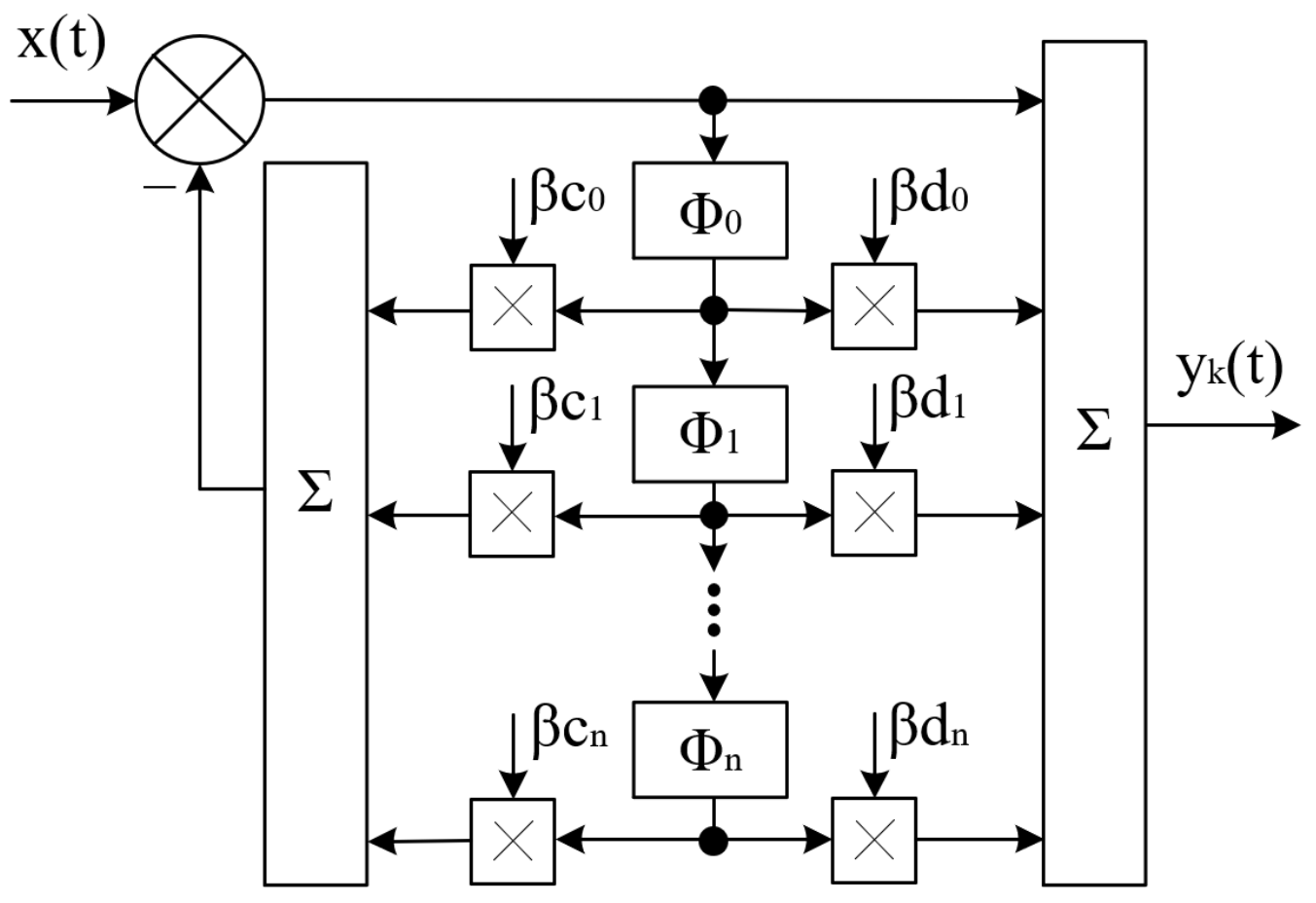

Рис. 1. Структура диагностической модели для k-ой контрольной точки

поиска параметрических дефектов непрерывных САУ. Идея предлагаемого алгоритма строится на совместном использовании методов диагностики и идентификации. Было определено, что лучшие результаты диагностирования обеспечивает модель на фильтрах Лагерра, включенных в обратную связь. При этом было рассмотрено применение фильтров, включаемых только в обратную связь, либо в прямой цепи. Цель данной работы - рассмотрение возможности применения динамических моделей на фильтрах Лагерра, построенных по канонической форме, и определение возможных преимуществ и недостатков такой диагностической модели.

\section{Описание алгоритма}

В данной работе для построения фильтров изоморфной динамической модели используется каноническая форма, которая имеет в своем составе каскадное соединение рекурсивной и нерекурсивной частей. Общий вид моделей для $n$ базисных функций $\Phi_{i}(t)$ представлен на рисунке 1. Здесь $x(t)$ - диагностический сигнал, подаваемый на вход модели и объекта; $y_{k}(t)$ - выходной сигнал изоморфной модели для $k$-ой контрольной точки; $\beta c_{1} \ldots \beta c_{n}$ и $\beta d_{1} \ldots \beta d_{n}$ - настраиваемые коэффициенты изоморфной модели для рекурсивной и нерекурсивной частей.

В работе рассматривается система ортогональных базисных функций на основе фильтров Лагерра, кото- рые реализуются с помощью следующих передаточных функций:

$$
\Phi_{0}=\frac{s}{s+b}, \quad \Phi_{i}=\frac{s-b}{s+b},
$$

где $b$ - изменяемый характеристический коэффициент фильтров Лагерра; $\Phi_{0}$ - передаточная функция для первого звена фильтра; $\Phi_{i}-$ передаточные функции последующих звенев.

Коэффициенты $\beta c_{i}$ и $\beta d_{i}$ получаются из контуров самонастройки диагностической изоморфной модели, которые строятся по градиентному методу. В качестве критерия близости сигналов объекта и модели был выбран квадрат ошибки. Таким образом, расчет коэффициентов $\beta c_{i}$ и $\beta d_{i}$ осуществляется согласно следующим выражениям:

$$
\begin{aligned}
& \beta c_{i}(t)=-a c \int_{0}^{t} e(t) u_{i}(t) d t, \\
& \beta d_{i}(t)=-a d \int_{0}^{t} e(t) u_{i}(t) d t,
\end{aligned}
$$

где $u_{i}(t)$ - функция выхода $i$-го базисного блока изоморфной модели, $e(t)-$ функция критерия близости объекта и модели, ac и ad - изменяемые коэффициенты для рекурсивной и нерекурсивной частей.

В предлагаемом алгоритме поиск параметрического дефекта в объекте осуществляется путем сравнения 


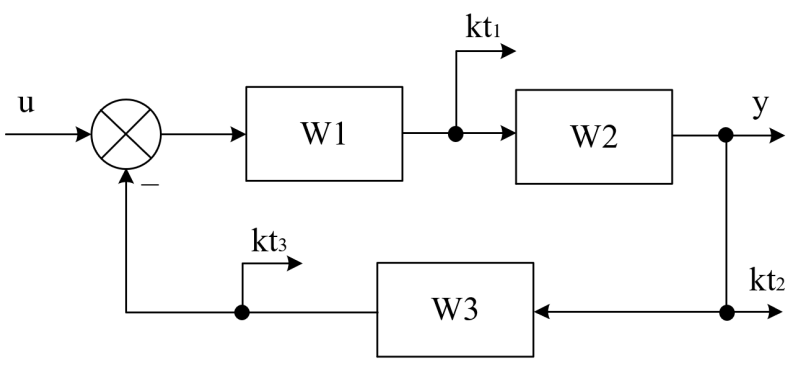

Рис. 2. Структура объекта диагностирования

векторов текущего технического состояния объекта с набором эталонных векторов дефектов, которые рассчитываются на этапе настройки диагностической модели. Векторы описывают одно из возможных дефектных состояний объекта с помощью своих параметров, которыми являются значениями коэффициентов $\beta c_{i}$ и $\beta d_{i}$. В данном случае, при правильной настройке диагностической модели, за определенное время эти коэффициенты стремятся к константе. Сравнение векторов осуществляется путем определения близости векторов друг к другу с помощью расчета диагностических признаков дефектов согласно следующему выражению:

$$
J_{j}=1-\left(\frac{(Y-N) \cdot\left(Z_{j}-N\right)}{\|Y-N\| \cdot\left\|Z_{j}-N\right\|}\right)^{2},
$$

где $Y$ - вектор параметров текущего состояния объекта диагностирования; $Z_{j}$ - вектор эталонных параметров $j$-ого рассматриваемого дефекта; $N$ - вектор параметров нормального (исправного) состояния объекта диагностирования. Данные три вектора составлены в совокупности по всему набору используемых контрольных точек. Значения диагностического признака лежат в пределах от 0 до 1. Если признак равен 0, то это означает полное совпадение векторов.

\section{Численные эксперименты}

В данной работе для проверки работоспособности предлагаемого алгоритма используется графическая интерактивная среда блочного моделирования Scilab/ Xcos. В качестве объекта диагностирования была рассмотрена структура САУ в типичном представлении: объект управления и элемент управления, охваченные отрицательной обратной связью. Его структура показана на рисунке 2 , где $u$ - входной сигнал объекта, $y$ - выходной сигнал, $k t_{1} \ldots k t_{3}$ - сигналы контрольных точек.

Блоки «W $1 »$, « $W 2 »$ и «W3» соответственно реализуют следующие передаточные функции:

$$
\begin{aligned}
& W 1(s)=\frac{b_{10}}{a_{11} s+1}=\frac{2}{s+1}, \\
& W 2(s)=\frac{b_{20}}{a_{21} s+1}=\frac{2}{2 s+1}, \\
& W 3(s)=b_{30}=1 .
\end{aligned}
$$

Для каждой контрольной точки объекта, которые устанавливались после каждого динамического блока, строится изоморфная модель, согласно рисунку 1. При проведении экспериментов значения коэффициентов $\beta c_{i}$ и $\beta d_{i}$ не всегда стремятся к определенному значению в конце времени моделирования, а могут совершать колебания вокруг постоянного значения. Поэтому значения коэффициентов рассчитываются по среднему значению последних $10 \%$ отсчетов.

Настройка изоморфных моделей проводилась согласно алгоритму, описанному в работах [8, 9]. Значение параметра $N$ (количества используемых базисных функций) определятся по порядку передаточной функции объекта диагностирования и выбирается равным ему или большим на одну или две единицы. Параметр $b$ определяет быстродействие фильтров Лагерра. Его лучше выбирать таким, чтобы переходные характеристики звеньев фильтра в общем соответствовали переходной характеристике объекта диагностирования. Параметры $a c$ и $a d$ в контурах самонастройки, которые в основном влияют на скорость работы модели, выбирались экспериментально. Таким образом, были выбраны следующие параметры изоморфных моделей:

$$
N=3, \quad b=0.27, a c=1.6, a d=-1.1 .
$$

Для проведения экспериментов по определению параметрических дефектов был определен набор векторов эталонных дефектных технических состояний объекта для каждого динамического блока. Набор состоит из 20 векторов для отклонений в значениях каждого параметра на $\pm 10 \%$ и $\pm 25 \%$. В качестве диагностируемых дефектов были рассмотрены отклонения параметров на $\pm 7 \%, \pm 14 \%$ и $\pm 20 \%$. Результаты прове- 
Таблица 1. Результаты диагностирования

\begin{tabular}{|c|c|c|c|c|c|}
\hline $\begin{array}{l}\text { Введенный } \\
\text { дефект }\end{array}$ & $\begin{array}{l}\text { Эталонный } \\
\text { дефект }\end{array}$ & $\begin{array}{l}\text { Диагностический } \\
\text { признак }\end{array}$ & $\begin{array}{l}\text { Введенный } \\
\text { дефект }\end{array}$ & $\begin{array}{l}\text { Эталонный } \\
\text { дефект }\end{array}$ & $\begin{array}{l}\text { Диагностический } \\
\text { признак }\end{array}$ \\
\hline $\mathrm{a}_{11}-7 \%$ & $\begin{array}{l}\mathrm{a}_{11}-10 \% \\
\mathrm{a}_{11}+10 \% \\
\mathrm{a}_{11}-25 \% \\
\mathrm{a}_{11}+25 \% \\
\mathrm{~b}_{30}-25 \%\end{array}$ & $\begin{array}{l}0.0000046 \\
0.0001478 \\
0.0001632 \\
0.0005246 \\
0.8578172\end{array}$ & $\mathrm{a}_{11}+20 \%$ & $\begin{array}{l}a_{11}+25 \% \\
a_{11}+10 \% \\
a_{11}-10 \% \\
a_{11}-25 \% \\
b_{30}-25 \% \\
\end{array}$ & $\begin{array}{l}0.0000128 \\
0.0000514 \\
0.0004605 \\
0.0010299 \\
0.8641984\end{array}$ \\
\hline$b_{20}-14 \%$ & $\begin{array}{l}\mathrm{b}_{20}-10 \% \\
\mathrm{~b}_{20}-25 \% \\
\mathrm{~b}_{20}+10 \% \\
\mathrm{~b}_{20}+25 \% \\
\mathrm{a}_{21}+25 \%\end{array}$ & $\begin{array}{l}0.0001425 \\
0.0013728 \\
0.0038591 \\
0.0084195 \\
0.6130306\end{array}$ & $b_{20}-20 \%$ & $\begin{array}{l}\mathrm{b}_{20}-25 \% \\
\mathrm{~b}_{20}-10 \% \\
\mathrm{~b}_{20}+10 \% \\
\mathrm{~b}_{20}+25 \% \\
\mathrm{a}_{21}+25 \%\end{array}$ & $\begin{array}{l}0.0003125 \\
0.0009807 \\
0.0066301 \\
0.0123166 \\
0.6263334\end{array}$ \\
\hline $\mathrm{a}_{21}+7 \%$ & $\begin{array}{l}\mathrm{a}_{21}+10 \% \\
\mathrm{a}_{21}-10 \% \\
\mathrm{a}_{21}+25 \% \\
\mathrm{a}_{21}-25 \% \\
\mathrm{~b}_{20}+25 \% \\
\end{array}$ & $\begin{array}{l}0.0000356 \\
0.0011907 \\
0.0012388 \\
0.0043481 \\
0.5787546\end{array}$ & $a_{21}-20 \%$ & $\begin{array}{l}\mathrm{a}_{21}-25 \% \\
\mathrm{a}_{21}-10 \% \\
\mathrm{a}_{21}+10 \% \\
\mathrm{a}_{21}+25 \% \\
\mathrm{~b}_{20}+25 \%\end{array}$ & $\begin{array}{l}0.0001125 \\
0.0004362 \\
0.0037589 \\
0.008177 \\
0.6288635\end{array}$ \\
\hline$b_{30}+14 \%$ & $\begin{array}{l}b_{30}+10 \% \\
b_{30}+25 \% \\
b_{30}-10 \% \\
b_{30}-25 \% \\
b_{20}+25 \%\end{array}$ & $\begin{array}{l}0.0000373 \\
0.0002362 \\
0.0017529 \\
0.0058223 \\
0.7333311\end{array}$ & $b_{30}-20 \%$ & $\begin{array}{l}b_{30}-25 \% \\
b_{30}-10 \% \\
b_{30}+10 \% \\
b_{30}+25 \% \\
b_{20}+25 \%\end{array}$ & $\begin{array}{l}0.0001554 \\
0.0004898 \\
0.0033458 \\
0.0062697 \\
0.7204141\end{array}$ \\
\hline
\end{tabular}

денного диагностирования представлены в таблице 1. Ввиду большого количества рассмотренных дефектов, в таблице приведена только их часть, а также сокращен список значений диагностических признаков.

Проведенный эксперимент по диагностированию параметрических дефектов показал, что предлагаемый алгоритм правильно определил все введенные в объект дефекты. Для оценки эффективности работы алгоритма вычислялась различимость дефектов, за которую была принята разница между минимальным значением диагностического признака для вектора эталонного дефекта объекта не являющимся искомым и искомым. Таким образом средняя различимость составила 0.0004374. Малое значение различимости объясняется тем, что при расчете использовались значения признаков дефектов одного и того же параметра, но с разной степенью его отклонения. Также была рассчитана различимость для параметров, то есть разница между минимальным значением признака для вектора эталонного дефекта параметра не являющимся искомым и искомым. В данном случае средняя различимость составила 0.7028456.

\section{Выво $\triangle \mathrm{b}$}

В работе представлен алгоритм диагностирования одиночных параметрических дефектов в непрерывных САУ с помощью изоморфных моделей, основанных на фильтрах Лагерра, построенных в канонической форме. По результатам проведенных численных экспериментов по диагностированию вводимых дефектов видно, что предложенный алгоритм позволяет с высокой различимостью определить параметрический дефект, и с меньшей различимостью определить направление изменения отдельного параметра. Это является определенным плюсом, так как не все существующие методы и алгоритмы диагностирования позволяют определить направление изменения параметров в объекте. Малая различимость параметрического дефекта относительно группы эталонов, соответствующих его изменению на разную процентную величину, позволяет уменьшить количество используемых эталонов до числа параметров объекта диагностирования. В этом случае поиск дефектов будет осуществляться с высокой различимостью (выше 70\% от всей шкалы значений диагностических признаков). Рассмотренный способ построения фильтров в канонической форме обеспечивает немного большие показатели различимости, чем при использовании только рекурсивной или нерекурсивных частей, которые были представлены ранее в работах $[8,9]$. Это можно объяснить увеличением размерности используемых векторов технического состояния объекта. При этом увеличивается сложность настройки самой диагностической модели за счет необходимости выбирать два коэффициента в контурах самонастройки. Необходимо отметить, что существует возможность уменьшения размерности наборов эталонных векторов де- 
фектов, потому что некоторые признаки дефектов для одного параметра имеют довольно схожие значения.

Преимуществом предлагаемого алгоритма является то, что для настройки изоморфных моделей ненужно знать точного строения объекта диагностирования. Этот факт позволяет избавиться от зависимости от большой априорно известной информации об объ- екте. Еще одним плюсом предложенной методики является отсутствие необходимости сложных расчетов для создания моделей, так как их настройка проводятся экспериментальным методом под конкретный заведомо исправный объект диагностирования. Это обстоятельство позволяет исключить ошибки диагностирования, связанные с неадекватностью используемой динамической диагностической модели.

1. Iserman R. Fault-Diagnosis Application. Springer, 2011.

2. Voronin V.V., Davydov 0.A. Local Area Network Failures Types, Consequences and Criticality Analysis. Second Russia and Pacific Conference on Computer Technology and Applications (RPC), 2017. P. 184-187.

3. Cheng C., Chen T. Robust adaptive diagnostic observers design for actuator fault dynamic systems. 11th IEEE International Conference on Control \& Automation (ICCA), Taichung, 2014. P. 360-364.

4. Шумский А.Е., Жирабок А.Н. Принятие решений при диагностировании нелинейных динамических систем непараметрическим методом. Автоматика и телемеханика. 2021. Н. 2. С. 111-131.

5. Sayed-Mouchaweh M. Fault Diagnosis of Hybrid Dynamic and Complex Systems. Springer, 2018.

6. Patton R.J., Frank P.M., Clark R.N. Issues of Fault Diagnosis for Dynamic Systems. Springer, 2000.

7. Rosenwasser E.N., Yusupov R.M. Sensitivity of Automatic Control Systems. CRS Press, Boca Raton, 2019.

8. Bloshchinskiy V.D., Shalobanov S.V. Finding Parametric Defects in Continuous Dynamic Objects Using Isomorphic Models on IIR Filters and Laguerre Filters. 2020 International Conference on Industrial Engineering, Applications and Manufacturing (ICIEAM). Publisher: IEEE, 2020, P. 1-5.

9. Блощинский В.Д., Шалобанов С.В. Влияние параметров контура самонастройки изоморфной динамической модели на различимость дефектов непрерывных САУ. Информатика и системы управления. 2019. Н. 4(62). С. 92-100.

с Блощинский Владислав Дмитриевич ( vladkms94@mail.ru ),

Шалобанов Сергей Викторович ( shalobanov@mail.ru ), Шалобанов Сергей Сергеевич ( shalobanov_ne@mail.ru ).

Журнал «Современная наука: актуальные проблемы теории и практики»

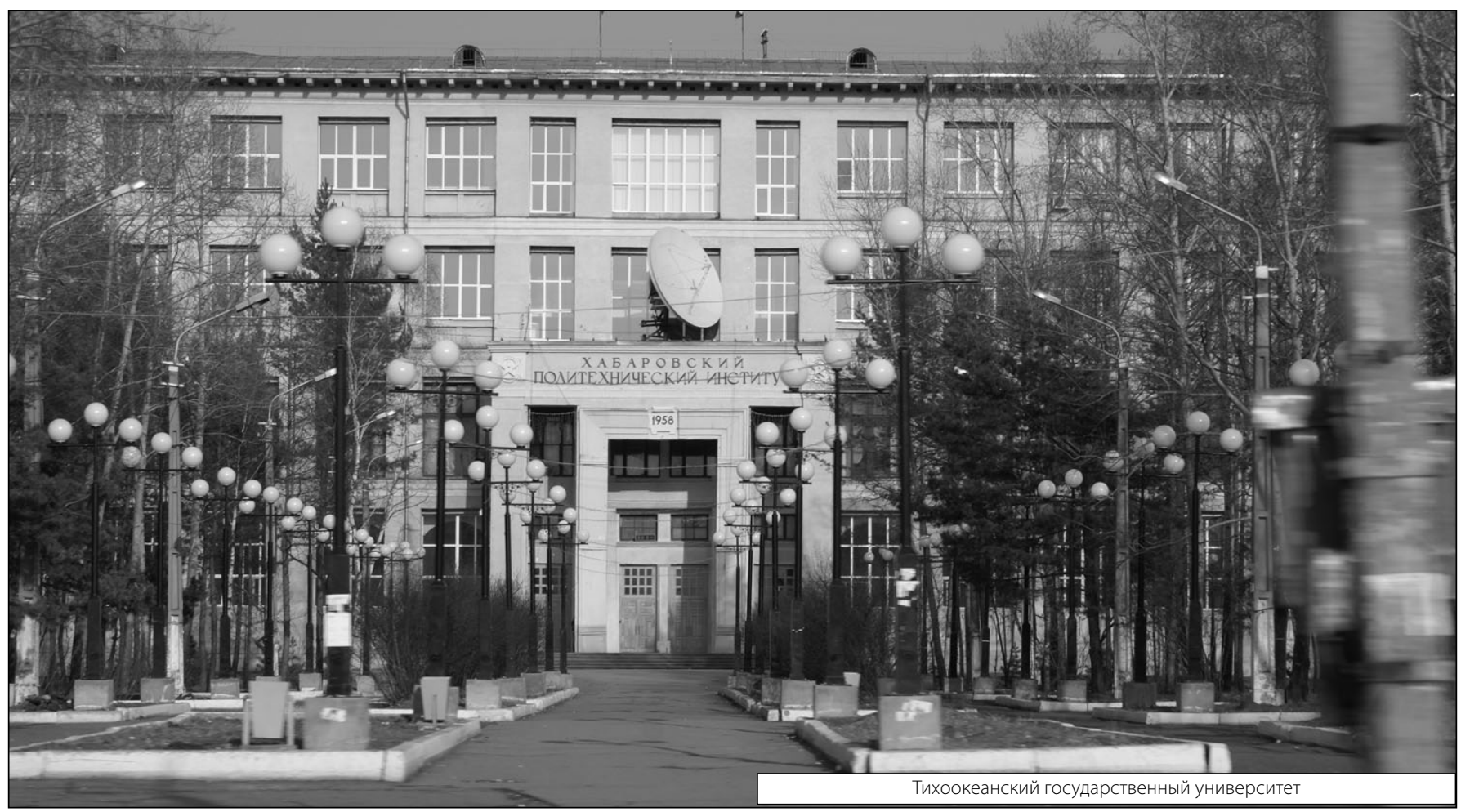

